\title{
Filigrane
}

Écoutes psychothérapiques

\section{De l'insensé au sens. Réflexions cliniques autour d'une pratique en milieu psychiatrique et carcéral}

\section{Aïcha Nora Dembri}

Volume 18, numéro 1, printemps 2009

Le corps. Sur le divan. Dans le fauteuil I

URI : https://id.erudit.org/iderudit/037721ar

DOI : https://doi.org/10.7202/037721ar

Aller au sommaire du numéro

Éditeur(s)

Revue Santé mentale au Québec

ISSN

1192-1412 (imprimé)

1911-4656 (numérique)

Découvrir la revue

Citer cet article

Dembri, A. N. (2009). De l'insensé au sens. Réflexions cliniques autour d'une pratique en milieu psychiatrique et carcéral. Filigrane, 18(1), 60-75.

https://doi.org/10.7202/037721ar d'utilisation que vous pouvez consulter en ligne. 


\title{
De l'insensé au sens. Réflexions cliniques autour d'une pratique en milieu psychiatrique et carcéral
}

\author{
aïcha nora dembri
}

\section{Introduction}

U ne activité clinique, actuellement moins intense, autour des interventions en pratique psycho-légale, m'a permis à mon insu de favoriser l'intégration d'une expérience clinique en milieu psychiatrique et légal à la fois saisissante et périlleuse.

À l'étape d'un bilan devenu maintenant possible et nécessaire, d'une pratique clinique d'approche psychodynamique, Filigrane me convie à partager les cycles et les étapes d'un travail théorique et clinique dans un espace institutionnel et thérapeutique où toute pratique était inféodée à une vision médicale-psychiatrique et judiciaire des soins.

Parmi les approches cognitivo-comportementales, très prisées, pour lesquelles l'intervention thérapeutique portait emphatiquement sur la correction des distorsions perceptives et la modification du comportement «déviant», faisait figure de «parent pauvre»l'approche psychodynamique consistant à donner du sens à l'insensé, à tisser des liens là où il y a discontinuité, à faire émerger le symbole délogé par le passage à l'acte.

Je me sentais à l'étroit parmi le succès grandissant des pratiques cognitivocomportementales en vogue, et celles-ci m'ont rendu ce terrain de pratique étranger et «étrange » à moi-même.

\section{Aléas d'une rencontre annoncée}

Au terme d'un remplacement en pédopsychiatrie et en quête d'un travail dans le même secteur, un collègue m'a proposé d'appliquer sur un poste de psychologue à l'Institut Philippe Pinel.

À priori, je n'avais pas d'intérêt pour le type de problématiques rencontrées à cet institut, où les actes d'une violence extrême s'arrimaient à des organisations psychopathologiques des plus complexes, mais j'étais contrainte par la nécessité de trouver un emploi et ma rencontre avec le monde psychiatrique et légal fut donc de nature plutôt fortuite.

Dans un premier temps, ma représentation de ces patients surprenants se butait à la confrontation de leur monde socio-relationnel immanquablement marqué par le 
sceau d'une violence délictuelle, destructrice et souvent insoutenable (sexuelle, physique, meurtrière...) mais aussi, de leur monde interne fréquemment coloré par la polymorbidité de leur pathologie (psychiatrique, organique, toxique, antisociale...).

Cependant, malgré ces apparences cliniques qui ne me conviaient pas de prime abord à l'exploration ni à la compréhension de la nature profonde d'un psychisme si perturbé, l'évocation de leurs tragédies aux consonances si douloureusement humaines a vite fait de capter mon attention et ma curiosité.

En effet telle mère infanticide faisait résonner en moi le drame de Médée, telle autre réveillait l'écho d'Agave, tel uxoricide (meurtre de la conjointe) ravivait en ma mémoire la passion possessive d'un Othello, tel matricide faisait revivre la haine meurtrière d'une Électre, tel fratricide le drame biblique d'un Caïn et Abel. Les exemples étaient ainsi à l'infini.

C'est donc cette figuration empruntée à la culture qui m'a servi de filtre et de fil d'Ariane pour m'approcher des couches profondes de l'activité psychique de ces identités morbides mais terriblement souffrantes.

\section{Cadre de soins et de prises en charge}

En regard des conditions sécuritaires au double plan des facteurs de risque de récidive et de la protection du public, toute intervention thérapeutique devait se soumettre au cadre médico-légal, dont l'institution dictait les règles. Cet impératif légal imposait aux soins la constitution d'une identité bicéphale formalisée, d'une part, par des traitements de type médico-psychologiques justifiés par la souffrance et les graves troubles psychiatriques et, d'autre part, par des interventions de type pénitentiaire justifiées par la perpétration d'un délit majeur.

Dans ce terreau clinique rébarbatif mais pourtant si fertile et prometteur, les actions thérapeutiques de type psychodynamique confrontées à cette dualité des soins se trouvaient dans un dilemme permanent: traiter la souffrance oui mais... Délit, incarcération, oui mais...

Cette ambiguitté clinique et cette oscillation ambivalente, loin de me rebuter, ont paradoxalement infléchi mon regard vers cette population si déroutante et ont ainsi provoqué, presque à mon insu, l'émergence des premiers constats cliniques.

\section{Confrontation au statut médico-légal et clinique des patients}

Les patients, sous mandat d'hospitalisation et de traitement non volontaire, demeuraient réfractaires à des interventions psychothérapiques.

Cette particularité de leur statut rendait d'emblée incongrue toute forme de prise en charge librement consentie.

Alors que leurs délits se caractérisaient par une violence primitive et fondamentale, leur psychopathologie se traduisait par des maladies mentales qui altéraient non seulement leur manière de raisonner et de juger, mais qui les livraient également aux marasmes de réactions pulsionnelles archaïques.

Avec ces problématiques aux confins de la déraison et de la destruction, qui abolissaient chez eux leur capacités d'être sentant (porteurs de sensation) et d'être 
pensant (porteur de sens), ainsi que leurs capacités d'être civilisés (créativité, échanges sociaux et humains préservés), ces patients demeuraient pour moi difficilement accessibles aux interventions de type psychodynamique (Dembri, 1997).

\section{Doutes et interrogations théorico-cliniques}

Alors que je m'interrogeais encore sur la pertinence d'intervenir avec ces patients selon une approche psychodynamique, je ne savais pas non plus quelle formulation théorico-clinique serait la plus à même de décrire les constellations psychiques complexes et compliquées de ces problématiques singulières : psychodynamique, cognitive, biologique, neurodynamique, anthropologique, sociologique...?

Dans ce même ordre de réflexions, je me demandais quelles configurations devaient avoir les interventions thérapeutiques pour concilier:

1. Ordonnance légale (dont les règles sont dictées par l'institution) et approches thérapeutiques,

2. Traitements pharmacologiques et psychothérapeutiques.

Dans l'optique du volet clinique qui me concernait tout spécifiquement, je me demandais si les interventions psychothérapeutiques devaient se réclamer d'approches :

1. Psychodynamiques classiques,

2. Psychodynamiques classiques avec aménagement du cadre et du contenu,

3. Innovatrices ou dissidentes,

4. Nouvelles.

\section{Patients entre univers externe et univers intrapsychique Constellation socio-familiale}

C'est mon intérêt, aussi littéraire soit-il, pour la tragédie humaine qui m'a permis de me rapprocher de ces patients et de me familiariser avec eux. Ce regard premier qui s'est posé sur eux, et que je considérais comme un «regard clinique », me révèle des individus prématurément façonnés par la violence agie et subie. Cette violence enracinée dans des traumas précoces leur a souvent dérobé leur intégrité physique ou a fait effraction dans leur psychisme en laissant d'indélébiles traînées mortifères.

Généralement, l'environnement dans lequel ils baignaient était perçu comme hostile et menaçant. C'est pourquoi, le monde qui les entourait était vécu comme malveillant et persécuteur, ce qui mettait alors leur existence en péril.

Ce sentiment de menace et de danger chronique légitimait pour eux toute attitude ou action violente visant à préserver leur intégrité.

Leur mode de relation se caractérisait par une emprise souvent tyrannique et le passage à l'acte était leur mode préférentiel d'expression.

Leurs délits portaient l'empreinte d'une rage destructrice, d'un raptus violent ou d'une destruction débridée. 


\section{Constellation psychique}

L'histoire de leur développement me dévoile des expériences précoces de frustrations et de gratifications chaotiques, marquées par des carences importantes dans les fonctions de maternage qui ouvraient sur des attachements instables et aberrants. La pauvre qualité de leurs attachements débouchait sur des liens objectaux erratiques et impétueux. (Dembri, 2003).

La rencontre avec l'altérité, mal assumée, révélait un narcissisme fragile ou pathologique qui ouvrait sur des investissements affectifs souvent de nature égocentrique ou morbide.

Leur organisation dynamique confirmait la présence de pulsions généralement mal liées révélant la présence réduite de pulsions libidinales au profit de pulsions agressives morbides. Ces éléments pulsionnels, s'accompagnaient perpétuellement de dysrégulation psycho-affective ou psycho-sexuelle.

Leurs perceptions étaient fréquemment dissociées ou fragmentées, leurs états traduisaient des angoisses de persécution, de morcellement ou de perte objectale intolérable. Les défenses archaïques se manifestaient sous forme d'identifications projectives et introjectives pathologiques, de déni, de clivage, de dissociation, d'omnipotence (Dembri, Lusignan et Marleau, 2004).

Au niveau de leur organisation cognitive et mentale, la pensée était de type concret ou opératoire concret. Le langage demeurait fruste et habillait très peu leurs pensées.

L'absence de rêves était parfois remplacée par des récits oniriques fragmentés ou par des cauchemars. Ces cauchemars saturés par des images violentes, où la mort et l'agression tenaient les devants de la scène, révélaient leur nature traumatique (corps démembrés et ensanglantés, personnages hideux violeurs ou cannibaliques...).

Comme ces patients avaient de la difficulté à se représenter mentalement leurs conflits psychiques, la pauvreté de l'élaboration par blocage de la symbolisation était retrouvée dans toutes les formes d'expression émotionnelle et personnelle. Cet aspect expliquait en grande partie leur difficulté à se représenter imaginairement ou à se raconter en récits imagés. Les fonctions cognitives dans leur registre symbolique étaient peu accessibles dans un premier temps.

Ces composantes qui concouraient à me révéler un espace intrapsychique aux contours mal délimités et aux contenus mortifères, mais paradoxalement d'une grande vitalité, m'avaient forcée à m'interroger sur la nature et la qualité du processus de subjectivation chez ces patients, mais aussi sur la nature de leur destructivité.

En effet, quelle compréhension et quelle place devais-je accorder à cette violence agie dans leur configuration psychique et en corollaire l'approche thérapeutique à adopter?

\section{Patients et passage à l'acte}

Plus souvent qu'autrement, le passage à l'acte visait l'anéantissement d'autrui et provenait de deux sources: 
1. Non délirante qui sous-tendait des comportements antisociaux ou pervers,

2. Délirante corrélative d'organisation psychotiques parfois très sévères.

L'analyse de leurs actes dévastateurs m'avait permis de constater que l'agir était relié à des souvenirs mémorisés et fixés de manière traumatique. Cette perception d'événements traumatiques aux évocations crues ou pauvrement imagées, était en lien avec des violences, des frustrations massives ou attachements perturbés vécus réellement dans l'enfance.

Le contre-investissement de ces fixations traumatiques était alors confié à des objets externes: personnes, drogues.... Ces objets étaient ainsi utilisés dans une relation d'agrippement, d'emprise fétichique, perverse... (Dembri, Lusignan et Marleau, 2004)

La compréhension clinique de leurs actes m'a laissé entrevoir qu'il y avait un déséquilibre entre la représentation mentale et l'acte lui-même. Ce déséquilibre était à l'origine d'une brèche dans leur organisation structurale. De cette faille dans leur structure, s'écoulait le monde interne et s'engouffrait le monde externe de ces patients. Ces deux espaces obligatoirement distincts étaient alors souvent confondus. C'est cet amalgame atavique qui expliquait, alors, comment l'instant externe du geste fatidique et leur réalité interne s'étaient retrouvés fusionnés.

Le passage à l'acte devenait ainsi une coïncidence tragique et funeste entre leur monde interne et la réalité extérieure désormais indissociables.

Cette faille structurale m'était apparue commune à tous ces patients et semblait caractériser les mécanismes reliés au passage à l'acte lui-même.

Ce constat clinique m'a permis de mieux saisir les enjeux psychiques d'une telle violence et m'a aidée à entrevoir l'esquisse d'une intervention thérapeutique possible et envisageable.

\section{Patients : espace intrapsychique revisité}

Leur difficulté ou incapacité à organiser des récits de vie pouvant être évoqués sous forme d'images symboliques ou figuratives m'a confirmé que la carence des expériences de gratification entravait assurément la constitution de solides images internes. Cette impasse psychique m'a révélé que l'élaboration mentale médiatisée par l'image était impossible pour ces patients. Elle m'a également indiqué chez eux, les défaillances de l'imagination appelée aussi «activité psychique imageante ou représentation imagée » (Tisseron, 1997).

Je pouvais donc supputer, en ce qui concerne leur fonctionnement psychique que la carence mentale et symbolique n'a pas permis l'instauration d'un espace psychique interne nécessaire à l'aménagement et à la transformation de la pulsion, de ses investissements et de ses vicissitudes.

L'édification d'un espace psychique devenait donc le cœur même de l'action thérapeutique à mettre en place avec ces patients.

Cependant, la construction de cet espace psychique imposait l'existence de conditions préalables au langage et dont l'image, l'imagination et la symbolisation en étaient les indispensables piliers. 


\section{Le corps du travail thérapeutique Bases théorico-cliniques}

Dans ce parcours labyrinthique et hasardeux où je valsais tantôt avec la «folie», tantôt avec la violence fondamentale, et plus souvent avec les deux, je me demandais comment formuler les assises théoriques et cliniques qui me permettraient d'identifier et de cerner les figures de la pulsion, les conditions et l'espace propice à son déploiement et à ses métamorphoses.

Il est généralement admis que la pulsion prend source dans le corps et qu'elle s'enracine dans les expériences sensorielles et sensori-motrices précoces (Anzieu, 1986; Tisseron, 1997). Cependant pour devenir représentable la pulsion doit bénéficier d'une aire propice à son éclosion. Cette surface d'expression de la pulsion qui est alors portée par l'imaginaire se voit délimitée par les interdits et habillée par les enveloppes psychiques (Anzieu, 1986, 1996).

Autrement dit la pulsion ne prend corps et ne se profile que grâce à ces deux modalités : l'interdit (notamment l'interdit du toucher) et l'enveloppe psychique.

La pulsion, dès lors contrainte par l'interdit, se contient, se différencie et se localise par l'enveloppe psychique. Cette contention, modifie et transforme la pulsion qui devient désir. Balisée par les interdictions morales et sociales, la pulsion qui se modifie en désir, tend éventuellement à se sublimer sous la poussée culturelle.

Nous savons également que les images s'enracinent dans le corps et se trouvent au carrefour de la sensori-motricité et de la vie pulsionnelle. L'image, fonctionnant alors comme premier contenant ou enveloppe psychique (Tisseron, 1997), revêt trois formes successives et complémentaires de symbolisation de l'expérience : sensori-affectivo-motrice, représentation mentale imagée et représentation verbale.

L'image s'avère alors être une opération de base indispensable du fonctionnement psychique déployée sur la sensori-motricité. Elle s'érige ainsi en enveloppe ou contenant psychique de la pulsion et devient une partie importante du processus de symbolisation (Tisseron, 1997).

\section{Objectifs thérapeutiques avec nos patients}

Je savais désormais que l'identité psychique de nos patients se diffusait et s'animait dans leurs agirs destructeurs. Le patient mettait en acte, en lieu et place du souvenir, ses affects et indices primaires (sensorialités, sensations) dans lesquels étaient encryptés les traumatismes réels et imaginaires. L'agir comme expression de l'affect et du désir se substituait ainsi à l'ordre symbolique de la parole.

Il m'a semblé donc, que le corps de l'action thérapeutique avec ces patients invitait non seulement à ce travail de contention et de sublimation de la pulsion destructrice, telle que figurée dans leurs passages à l'acte, mais également à rétablir chez eux les conditions intrapsychiques qui les réhabiliteraient dans l'ordre symbolique comme être «sentant et pensant». 
Les modalités de cette action thérapeutique devraient alors cibler la restauration et la revalorisation de l'image, de l'imagination, de la symbolisation et de la sublimation. Ces piliers qui soutiennent de manière complémentaire, l'édification tant externe (enveloppe) qu'interne (contenu) de l'appareil psychique devraient être au centre du transfert. La vie psychique qui en résulterait deviendrait aussi métamorphosable à l'intérieur de la relation transférentielle.

\section{Modalités des interventions thérapeutiques}

Par contre, avec ces patients «lourdement handicapés » sur le plan du symbolique et de la signification, le fait de centrer d'emblée le cœur de l'intervention thérapeutique sur le langage et la parole, comme le voulait l'approche psychodynamique, m'est apparu peu valable et voire même une erreur à éviter. Par conséquent, j'avais alors exploré l'idée de considérer, dans un premier temps, des avenues thérapeutiques avec des formes de représentations plus appropriées à ces patients.

De plus, avec ces personnes portées par des angoisses archaïques et aux narcissismes pathologiques, il m'a paru aussi, que tout espace de rencontre thérapeutique devrait être médiatisée. En effet, l'introduction d'un «tiers », tel le «bouclier de Percée», qui déjouerait le «face à face» mortifère, inciterait à une diminution de l'angoisse et paverait alors la voie à une parole porteuse de sens et d'interprétation mieux accueillie.

$\mathrm{Ne}$ serait-il pas alors judicieux ou plus approprié d'inclure des modes ou moyens d'expression à travers lesquels les patients me révèleraient les figures encryptées de leurs traumatismes, de leurs affects et de leurs désirs?

Puisque c'est dans l'agir et dans l'acte que les patients se donnaient vie et que leurs émotions prenaient corps, ne devrais-je pas utiliser en premier lieu, tant dans les interventions individuelles que de groupe, des techniques qui favoriseraient non seulement ce moyen «agi » d'expression mais qui feraient également fonction de tiers?

J'avais pour ma part opté pour :

1. l'acte graphique: séances de dessin en rencontre individuelle ou en groupe d'art-thérapie,

2. l'acte ludique: groupe de symbolisation et de psychodrame.

$\mathrm{Au}$ niveau institutionnel, le tiers représenté par le médical (psychiatre) était symbolisé dans l'aire thérapeutique par l'action pharmacologique. Ce «médicament-tiers » en abaissant le taux invalidant d'angoisse, avait permis une liaison efficace de la pulsion et de sa représentation verbale.

Dans le contexte du travail avec ces patients, la médication devenait ainsi une partie intégrante des moyens désignés pour supporter et consolider la construction du réseau symbolique des interventions mises en place.

\section{Cadre thérapeutique}

La dissonance qui existait, d'une part, entre le niveau et le degré de fonctionnement symbolique de ces patients et, d'autre part, la nature encore obscure de leurs 
pulsions confirmait non seulement l'absence de liaison entre ces pulsions et leurs représentants psychiques, mais également le mauvais enchaînement entre le fonctionnement symbolique de ces patients, l'utilisation de la parole et du langage comme mode d'expression et de communication.

Cette discordance intrapsychique les exposait alors au risque de succomber au déferlement incontrôlable de leurs pulsions. Le passage à l'acte meurtrier de nos patients illustrait bien la violence dévastatrice de ces excès pulsionnels par défaut de symbolisation.

On sait, que si l'interdit symbolique signale les dangers internes liés à la pulsion d'attachement, d'aggripement et d'emprise, les interdictions qui portent sur des conduites envers les objets extérieurs sont subordonnées au principe de réalité et aux règles morales et sociales (Anzieu, 1986, 1996).

Ces modalités opératoires forcent la pulsion à s'adapter à la réalité du monde extérieur. Comme je l'ai précédemment mentionné, on sait que la pulsion entravée par l'interdit et notamment l'interdit du toucher la force à se contenir, à se différencier et à se circonscrire par l'enveloppe psychique. Les interdictions comme modalité socio-culturelle, la poussent alors à «s'humaniser et à se socialiser»

Les interdits et les interdictions sont donc des corrélats essentiels à l'existence et à la sublimation de la pulsion.

Quel cadre thérapeutique devrais-je donc mettre en place et qui serait susceptible de convenir à un travail sur la pulsion et ses vicissitudes?

Pour ces patients, marqués par le sceau de la transgression et ayant porté atteinte à l'intégrité physique ou psychique d'autrui, dominés par des pulsions destructrices et par des liens d'emprise pathologiques, il m'est apparu essentiel d'énoncer les règles de fonctionnement du cadre thérapeutique sous forme d'interdits. Entre autres, lors des groupes de symbolisation ou de psychodrame, il était interdit de toucher autrui, il était également interdit de jouer les délits perpétrés ou toute autre forme de transgressions universelles. Le geste meurtrier était seulement mis en mot (Dembri, 1997).

$\mathrm{Au}$ niveau institutionnel, ces «interdits » étaient doublés par les règlements légaux qui délimitaient le cadre institutionnel et définissaient les conduites légales et sociales à suivre.

Ainsi si le cadre thérapeutique veillait à instaurer les interdits symboliques, l'institution devenait garante des interdictions en conformité avec le monde extérieur.

\section{Contenus thérapeutiques}

Il est important de mentionner que le dispositif thérapeutique mis en place ne reposait pas uniquement sur notre seule action, mais a nécessité la participation directe ou indirecte de professionnels, membres du personnels paramédical, responsables administratifs, etc. Cependant, comme l'objectif de cet article ne consiste pas à décrire de façon détaillée l'armature de ce système thérapeutique, je renvoie le lecteur à mon article publié dans Filigrane et qui explique en détail et 
avec vignettes cliniques à l'appui, l'organisation des soins mis en place avec ces patients (Dembri, 1997).

Je vais par contre, esquisser les grandes lignes cliniques du travail réalisé avec ces patients et évoquer succinctement le cadre et le contenu du travail thérapeutique accompli dans chaque type d'activité. Les patients ont participé à l'ensemble des activités thérapeutiques mises en place.

\section{Thérapie individuelle par le dessin et groupe d'art-thérapie}

Cette activité graphique avait pour but la restitution et la restauration de l'image comme enveloppe et contenu psychiques. Ces images psychiques tout en me révélant un état de la psyché de ces patients s'étaient avérées un puissant levier de changement interne. Les thérapies organisées autour de cette activité avaient pris la forme d'une expérience de communication interactive qui, tout en modifiant l'état psychique de l'interlocuteur, avait mis en place les schèmes psychiques de transformation (Dembri, 2003 ; Dembri, Lusignan et Marleau, 2004).

Le cadre thérapeutique et l'attention du thérapeute correspondaient à une forme d'enveloppe et avaient été considérés comme des équivalents symboliques.

Les images (dessins) issues de cette activité m'avaient renseignée:

1. sur la nature et la qualité des sensorialités primaires, sur les expériences traumatiques précoces de non-communication et sur l'évolution psychosexuelle et psycho-affective de ces patients (Dembri, 2003);

2. sur la façon de soutenir et d'aider ces patients dans leur évolution mentale;

3. m'avaient guidée vers les avenues à emprunter pour mener les patients d'une expression des affects vers leurs représentations mentales.

\section{Groupe de symbolisation: mythe, conte et masque}

Activité thérapeutique essentiellement basée sur le jeu symbolique, les thématiques des groupes de symbolisation (mythe, conte et masque) étaient dans un premier temps proposées par le thérapeute dans le but d'aider les patients à instaurer, via l'activité fantasmatique du thérapeute, un contact imaginaire et symbolique afin d'arriver ultimement par cette «prothèse psychique» à une activité fantasmatique qui leur serait propre (Dembri, 1997).

Le mythe: On sait, que le mythe met en scène un récit historique réel ou fictif dans lequel se projettent certains conflits individuels ou certaines structures en lien avec des complexes familiaux. Il est aussi avéré que, dans le cas de traumatismes ou d'actes destructeurs, les événements, les souffrances de l'âme, ou les sentiments ne peuvent être restitués à l'état brut. Ces événements tragiques doivent donc être préalablement pétris, accommodés et sublimés.

Dans le contexte des délits perpétrés par ces patients, l'utilisation des mythes m'a paru d'une importance capitale. Le mythe, comme patrimoine de l'humanité, m'a offert un récit d'événement dramatique prédigéré par la culture. Cette forme de figuration culturelle m'avait aidée à contourner la réalité brutale et crue du délit commis par ces patients et de trouver ainsi un sens symbolique au geste réel. 
Cet aspect régulateur de la transgression et de l'interdit m'avait autorisée à aborder ces deux dimensions dans leur registre symbolique et universel. Cette utilisation des mythes avait ainsi encouragé un travail thérapeutique de maturation et de maturité. Elle avait aussi favorisé l'instauration de la fonction morale alliée du surmoi et dépositaire des valeurs humaines.

Le conte: Il concerne habituellement les zones les plus primitives du psychisme. Il interroge la question-énigme sur les origines (vie), la différence des sexes et des générations, le passage de l'Enfance à l'âge Adulte, la relation entre le Désir et sa Réalisation, la menace de la Maladie et de la Mort (finitude). Le conte s'avère donc à la fois le récit d'un dérèglement et une réponse à ce dérèglement (Kaës et al., 1996).

Avec ces patients le conte m'avait permis, outre sa fonction symbolique, de mesurer le processus de métamorphose du corps et de son image, de l'utiliser comme processus de passage pédagogique, et de transformation sociale et culturelle.

Le masque : Il accompagne l'humain dans son histoire individuelle et sociale. En thérapie le masque interroge l'image du sujet, sa relation au social. Le masque révèle aussi le geste chargé de fantasme et le sens qui s'y cache.

L'utilisation du masque avec ces patients en thérapie avait favorisé un travail de « démasquage » restructurant mais il avait permis aussi de baliser et de marquer les points de conflits au niveau personnel et relationnel. Le masque avait autorisé un travail sur l'identité, sur l'étrange et l'étranger en soi et à soi (Vacheret et al., 2002).

Le psychodrame: J'avais ciblé le corps (moi corporel, corps imaginaire) comme objet de travail avec ces patients. La structure et l'organisation rythmique des séances avaient favorisé le processus de représentation et de son enveloppe psychique. L'acte de jouer avait encouragé un travail sur la transformation de la pulsion dans l'ici et maintenant (Dembri 1997; Kellerman, 2007).

La parole source de sens et d'interprétation, en introduisant une dimension symbolique avait ouvert sur un travail de transformation du réel en encourageant l'activation des processus associatifs.

\section{Réflexions et interrogations cliniques}

Au terme de ce travail qui s'était échelonné sur plusieurs années, l'évolution surprenante de certains patients, la métamorphose insoupçonnée de d'autres ou la crispation pathologique de tels autres, m'emmène à faire le point sur l'ensemble du dispositif thérapeutique qui avait été mis en place avec eux.

Avec ces patients qui pendant un temps avaient défié ou mis en doute mes paradigmes théoriques et cliniques, je peux désormais avancer les constats suivants.

En premier lieu, en regard des exigences médicales, j'ai pris conscience de combien la dimension pharmacologique pouvait être un agent adjuvant dans l'élaboration des conditions thérapeutiques pour une intervention optimale sur les composantes symboliques lourdement détériorées de ces patients. 
Filigrane, printemps 2009

Au niveau des conditions institutionnelles, la fonction incontournable du cadre légal et carcéral, loin d'être une entrave au travail psychodynamique, s'est avérée, en rétrospective, un puissant agent de délimitation et de structuration des contraintes morales et socio-culturelles.

Ces contraintes ont contribué non seulement à brider ces pulsions anarchiques et dévastatrices mais aussi à les confronter à l'épreuve de réalité du milieu ambiant.

Par cet aspect, le cadre institutionnel a propulsé l'émergence d'espaces de médiations indispensables entre la réalité psychique interne et la réalité externe du milieu social.

En d'autres termes, si le cadre institutionnel a contribué, en doublant les règlements thérapeutiques, à colmater la faille inhérente à leur fonctionnement structural et à mettre un frein à l'omnipotence des patients, il a également jugulé l'émergence ou l'expression d'un sentiment de toute-puissance chez les thérapeutes.

Exemple clinique: un patient qui a été acquitté pour cause de maladie mentale, d'un acte fratricide, s'évertuait à expliquer à son équipe traitante dont je faisais partie qu'il n'avait pas à suivre tous les traitements pharmacologiques ou psychothérapeutiques qui lui ont été proposés dans le cadre de son statut légal et médical, puisque son geste était de la légitime défense et qu'il n'était pas «fou».

Lors d'une séance de psychodrame, il voulait rejouer le genre de rapport abusif qui existait entre son frère et lui-même pour nous convaincre que son geste s'inscrivait bel et bien dans un acte de légitime défense. Alors qu'une réalité tout autre s'est dégagée du jeu, tel que proposé par le patient lui-même, ce dernier interrompit brutalement son jeu considérant que nous (les thérapeutes) voulions le rendre malade pour confirmer qu'il était «fou». Je lui ai alors demandé ce que signifiait pour lui "être fou». Le patient me répondit: "Si je suis fou, alors ce n'est pas de la légitime défense. C'est que j'ai tué un innocent et cet innocent est mon frère..."

Depuis lors, le patient fut convaincu que l'ensemble de l'équipe traitante s'était ligué contre lui pour le rendre malade. Il abandonna tous ses traitements et refusa de s'alimenter au point de mettre en danger sa santé physique. Des mesures médicales draconiennes furent prises pour rétablir son état physique. Le patient a alors porté plainte auprès de l'institution contre l'ensemble des professionnels, médecin, moi-même, infirmière, etc. alléguant le fait qu'il était maltraité et qu'on voulait nuire à sa santé physique et mentale.

Le patient fut donc rencontré personnellement, ainsi que les différents professionnels, afin de vérifier les différents points de vues. Comme la plainte ne fut pas retenue, il a été suggéré que le patient reprenne ses multiples traitements. Je me suis objectée à sa réintégration en thérapie tant et aussi longtemps que le patient ne soit pas rencontré par moi-même et en présence des instances institutionnelles pour lui expliquer le sens porté par ce "passage à l'acte» qui s'inscrivait dans 
une répétition du geste délictuel pour lequel il était traité. Pour éviter toute répétition compulsive ultérieure, j'avais souhaité que l'espace thérapeutique puisse être réparé et restauré sous le regard institutionnel avant toute reconduction de son suivi. Ce qui fut fait.

Après cette action l'implication et l'évolution du patient dans l'ensemble de son traitement furent exemplaires et son évolution surprenante. »

Sur le plan du travail thérapeutique proprement dit, l'utilisation de l'imagination, de la rêverie, et du fantasme, a encouragé la liaison entre les images et leurs enveloppes psychiques dans un ordonnancement logique et significatif. Cet agencement autour de l'intrication de la pulsion avec ses représentants psychiques a contribué à l'édification de la jonction reliant le langage à sa fonction symbolique.

Avec ces patients, ce travail autour du sens symbolique et de la signification a favorisé la rencontre inexorable et souvent mal anticipée avec les dangers internes reliés aux pulsions destructrices qui les habitent.

Dans ce parcours d'un travail thérapeutique laborieux, l'attention, l'intérêt et les interactions constants des thérapeutes en substituant au fonctionnement mental entravé, et voire même altéré, des patients un fonctionnement mental plus associatif, porteur de sens et de significations, ont tissé les liens indispensables à une continuité de l'expérience affective.

Avec ces problématiques complexes aux confins de la déraison et de la destruction, il m'est apparu impératif dans mes interventions de porter une attention toute spécifique à la pulsion et ses versatilités, en vue de détecter et de décrypter à travers ses transfigurations les nombreux scénarios qu'elle met en scène.

En accord avec ce qui est avancé, et bien que je n'avais pas, comme je l'aurais souhaité, porté à cet aspect une attention assidue, j' avais remarqué lors des séances de groupe (psychodrame, symbolisation, art-thérapie), combien la rencontre imprévue et saisissante de plusieurs espaces psychiques pouvait concourir au surgissement d'un espace psychique commun. Cet espace psychique collectif et partagé devenait une puissante matrice fantasmatique sur laquelle les patients pouvaient s'appuyer et qui portait en elle les germes propices à l'éclosion d'un espace psychique individué. Le travail de groupe et en groupe m'apparaissait ainsi porteur d'expériences émotionnelles structurantes, réparatrices, transformatrices et voire même créatrices.

Réhabiliter chez ces patients lourdement atteints leur statut d'être «sentant et pensant», suppose donc l'existence d'une vie psychique inconsciente métamorphosable à l'intérieur d'une relation et d'un espace transféro-contre-transférentiel (Kristeva, 1993).

Pourtant, chez certains patients, tissées dans ces relations précoces perturbées et se caractérisant par un moi marqué par de graves distorsions, certaines particularités de leur fonctionnement psychique semblaient relever d'une essence biologique. En effet, ces patients utilisaient une forme singulière de remémoration pour rendre compte d'une qualité d'émotions dissociées et qui s'avérait non communicable psychiquement. 
Cette modalité originaire d'évocation, à partir de rythmes, de mouvements corporels, de sensations épidermiques, sonores, visuelles, et qui porte les germes du sentiment de continuité de soi, est considérée comme un fonctionnement présymbolique à forte coloration sensorielle et somatique (Nicolo et Strinati, 2007).

Cette mémoire d'évocation dite préverbale était liée, chez ces patients, aux expériences traumatiques précoces et emmagasinée dans des images sensorimotrices plutôt que dans des représentations verbales. Ce fonctionnement préverbal qui rend compte d'expériences sensorielles et corporelles que les neuropsychanalystes nomment «mémoire procédurale » s'avérait pathogène ou traumatique et paraissait non décodable psychiquement. Cette «mémoire procédurale» mettait cependant en évidence, la façon pathogène d'interagir du patient dans ses rapports à l'autre. Ce fonctionnement morbide, s'il était non décodable psychiquement, était par contre repérable dans leurs comportements agis.

Quelle pourrait être alors la nature de cette parole porteuse de sens et d'interprétation qui se confondrait, ferait écho ou entrerait en résonance avec les fatalités présymboliques de ces patients? Quelle avenue cette parole interprétative devraitelle emprunter pour s'infiltrer jusqu'à leur quintessence biologique et la transformer ou pour décoder psychiquement ces liens pathologiques et pathogènes qui enchaînent de façon mortifère le patient à son environnement socio-affectif et relationnel?

Ces constats cliniques nous poussent dans le cadre de patients lourdement perturbés, à reconsidérer ou «personnaliser» le travail thérapeutique en réinterrogeant nos espaces et relations transféro-contre-transférentiels, en modifiant ou en inventant de nouveaux modèles théoriques plus conformes à cette néo-réalité psychopathologique (Kristeva, 1993).

À cet égard, je pense que les avancées en neurosciences et les travaux en neuropsychanalyse permettront d'affiner la compréhension théorique et clinique de certaines facettes de la vie psychique tel que la compréhension du substrat neurobiologique et neuropsychanalytique de l'attachement et de ses avatars, par exemple.

Grâce aux percées en neurosciences et en neuropsychanalyse, ces nouvelles compréhensions favoriseront la création d'approches thérapeutiques qui permettront d'intervenir et de modifier chez ces patients dans un espace et une relation transféro-contre-transférentiels les parcelles délétères de la vie psychique qui entravent pernicieusement l'évolution de leur processus subjectivant vers un destin intime et humanisant.

\section{Réactions-transféro-contre-transférentielles}

Au plan de l'environnement institutionnel, j'avais été persuadée pendant un certain temps qu'il me serait difficile d'intégrer cet aspect «bicéphale» du travail. Le caractère omniprésent de la sécurité, tant au plan humain (gardes de sécurité) qu'instrumental (portes qui se ferment automatiquement, bouton d'urgence...) 
m’apparaissait jusqu'à un certain point «paralysant». Mais étrangement, j' ai à mon insu et avec même une certaine facilité absorbée cette «ambiance coercitive».

Exemple clinique : En fait, plus qu'un exemple clinique ce fait, plutôt cocasse en soi, éclaire avec intensité l'omniprésence des mesures sécuritaires.

Lors d'une séance de groupe, nous étions tranquillement assis à écouter une patiente décrire son thème de jeu lorsque brutalement trois colosses firent irruption dans la salle de thérapie.

Ces «cerbères » vigilants et attentifs à notre sécurité furent décontenancés de nous voir tranquillement assis alors qu'ils étaient prêts à un assaut «mémorable».

L'énigme fut résolue quand nous avons découvert que l'intervenant qui m'accompagnait dans cette thérapie s'est malencontreusement appuyé sur le bouton d'urgence sans fil qui se trouvait logé dans la poche arrière de son pantalon. Ce contact «non prémédité a actionné le système d'alarme continue dans les locaux des gardes de la sécurité. Cette alarme continue, signifiait en terme de code de sécurité: personne en danger extrême et immédiat !

Parce qu'elles contiennent une mémoire présymbolique encryptée des traumas et des défenses mis en jeu, les formes sensori-motrices précoces, caractérisant tant le fonctionnement de certains patients en thérapie individuelle et tout particulièrement en thérapie de groupe, exigeaient des thérapeutes un surplus de travail psychique.

L'aide et la mise à distance mentale, que nous allions comme thérapeutes chercher auprès de nos collègues ou d'autres professionnels au sein ou en dehors de l'institution, fut indispensable et je pense même salutaire, pour les thérapeutes dans leur ensemble.

Lorsque les patients se confrontaient à leur destructivité et à ses conséquences, la souffrance intolérable qui les submergeait alors, ainsi que les impulsions suicidaires qui s'y greffaient, ont éveillé mes craintes et mes sentiments de doute quant à mes capacités à les accompagner avec prudence et contenance dans cette étape critique et «insoutenable» de leur parcours.

À cet égard l'environnement institutionnel dans sa globalité, (mesures de sécurités, mesures de soins d'urgence, surveillance médicale étroite...) était une condition qui m'a profondément aidée à consolider mon assurance et à apprécier la nature et la qualité de mon fonctionnement psychique et relationnel dans ces périodes de hautes tensions émotionnelles.

La violence des femmes m'est apparue plus troublante et plus déroutante. Leur destructivité avait quelque chose de sacrificiel et c'est face aux femmes que j'ai le plus souvent, comme thérapeute, approchée et éprouvée l'état de peur instinctive, d'anéantissement et de mort. Cet aspect a bouleversé mes croyances en ce qui concerne le Féminin en général et le Féminin «sacré» en particulier.

Étrangement, et contrairement à certains de mes collègues, tout au long de mon travail clinique qui s'est étalé sur plusieurs années, je n'ai jamais eu d'expériences 
négatives ou traumatiques avec ces patients, expériences qui auraient porté atteinte à mon intégrité physique ou psychique.

En rétrospective, je crois qu'ayant développé un «certain goût» pour le tragique et la dramaturgie humaine (ce qui explique fort probablement mon orientation vers des techniques thérapeutiques tel que le psychodrame), j'ai pu, sans crainte certes mais avec précaution, m'approcher et me placer suffisamment au bord de ce fonctionnement archaïque pour être en relation et en communication interpsychique avec ces patients considérés «dangereux » mais pourtant si terriblement humains.

Il n'en demeure pas moins, qu'une certaine prudence ou vigilance instinctive et atavique me servait de baromètre et de frein pour moduler et ajuster la distance relationnelle entre ces patients et moi-même afin de conserver cette zone de confort et de sécurité indispensable pour que ces résonances interpsychiques puissent advenir.

Bien que la réflexion qui suit puisse faire l'objet en soi d'un autre article, je pourrai néanmoins énoncer que le dispositif thérapeutique mis en place fut sérieusement mis à risque ou compromis institutionnellement lors d'un changement de direction et d'administration. Cette expérience fut pour moi plus pénible et plus «éprouvante» que celles que j'ai vécues avec les patients.

En effet, de par les enjeux financiers et administratifs, la décision de s'orienter vers des traitements plus pharmacologiques et des interventions de type plus «carcéral» m'a forcée à adopter une position éthique et faire un choix professionnel difficile et douloureux. Cette décision, engendrée par la souffrance et dans la solitude mais mûrement réfléchie, fut le renoncement ou le sacrifice consenti par moi, pour conserver et préserver une liberté de penser et un espace de créativité indispensable à mon «bien-être psychique et intellectuel».

Si le travail avec ces patients m'a autorisée à approcher cette ombre ou cette face «sombre» qui hante chaque humain, à apprivoiser la violence et les peurs traumatiques, il a aussi redirigé mon intérêt vers une compréhension clinique plus poussée de la pulsionnalité infantile et du destin des pulsions chez l'adolescent en quête de subjectivité.

\section{Conclusion}

Alors que je pensais à priori qu'une institution à l'identité double, psychiatrique et carcérale, pouvait être inconciliable avec des interventions de type psychodynamique, en mesurant les étapes engagées dans ce travail avec des patients en désertification psychique, je me demande en fait, si ce travail n'a été précisément concevable et réalisable que grâce à ce profil bicéphale de l'institution.

En effet, n'aurait-il pas été périlleux d'entreprendre une telle «aventure» dans une institution essentiellement médicale ou essentiellement carcérale ? Ce travail aurait-il été simplement possible ou envisageable?

Bien que l'institution où j'ai travaillé avait tendance à privilégier les interventions qui visaient un apprentissage cognitivo-comportemental en vue de corriger 
l'acte déviant, dans le cadre du travail que j'ai effectué, elle m'a néanmoins autorisée et encouragée à apprendre à jouer, à imaginer et à inventer avec ces patients en lieu et place de détruire. C'était m'autoriser à apprendre avec eux, non seulement à renoncer à leur destructivité, mais également à transmuer l'effroi et l'épouvante aliénants surgis des abysses insondables du trauma en créativité émancipatrice.

aïcha nora dembri

90, berlioz appt 1002

île des sœurs

verdun

qc h3e 1 n1

noradembri@vdn.ca

\section{Bibliographie}

Anzieu, D., 1986, Une peau pour les pensées, Clancier-Guenard, Paris.

Anzieu, D., 1996, Créer, détruire, Dunod, Paris.

Dembri, A. N., 1997, Agir et créativité dans des groupes de psychodrame et de symbolisation pour patients homicides, Filigrane, 6, 98-110.

Dembri, A. N., 2002, Femmes immigrantes, violence et santé mentale: solutions instrumentales ou institutionnelles? Actes du Colloques du Centre d'études appliquées sur la famille. Université Mc Gill, immigration et métropole (volet 4$)$.

Dembri, A. N., 2003, Entre Agave (violence meurtrière et délirante) et Médée (violence meurtrière vindicative), à propos de la violence instinctuelle et des troubles d'attachements chez les mères filicides, Psychologie clinique, $\mathrm{n}^{\circ} 16,125-138$.

Dembri, A. N., Lusignan, R., Marleau, J. D., 2004, Violence ou troubles sexuels : aspects pervers comparés chez de jeunes patients. Forensic. $N^{o}$ spécial : psychiatrie et violence, mars, 23-30.

Kaës, R., et al., 1996), Contes et divans : médiations du conte dans la vie psychique, Dunod, Paris.

Kellerman, P. F., Hudgins, M. K., 2001, Psychodrama with Trauma Survivors, Jessica Kingsley Publishers.

Kristeva, J., 1993, Les nouvelles maladies de l'âme, Fayard.

Nicolo, A. M., Strinati, E., 2007, Transmission du traumatisme et défenses transpersonnelles dans la famille, Cahiers critiques de thérapie familiale et de stratégie de réseaux, vol, 1, n 38, 61-79.

Tisseron, S., 1997, Psychanalyse de l'image, Dunod, Paris.

Vacheret, Cl. et al., 2002, Pratiquer les médiations en groupes thérapeutiques, Dunod, Paris. 